Cahiers $d u$ MONDE RUSSE

\section{Cahiers du monde russe}

Russie - Empire russe - Union soviétique et États indépendants

$44 / 4 \mid 2003$

Varia

\title{
Reinhard Müller, Menschenfalle Moskau
}

\section{Malte Griesse}

\section{OpenEdition \\ Journals}

Édition électronique

URL : https://journals.openedition.org/monderusse/4116

DOI : 10.4000/monderusse.4116

ISSN : $1777-5388$

\section{Éditeur}

Éditions de l'EHESS

\section{Édition imprimée}

Date de publication : 1 octobre 2003

Pagination : 742-748

ISBN : 2-7132-1833-0

ISSN : $1252-6576$

\section{Référence électronique}

Malte Griesse, «Reinhard Müller, Menschenfalle Moskau », Cahiers du monde russe [En ligne], 44/4 | 2003, mis en ligne le 19 juin 2009, consulté le 03 septembre 2022. URL : http://

journals.openedition.org/monderusse/4116; DOI : https://doi.org/10.4000/monderusse.4116

Ce document a été généré automatiquement le 3 septembre 2022

Tous droits réservés 


\title{
Reinhard Müller, Menschenfalle Moskau
}

\author{
Malte Griesse
}

\section{RÉFÉRENCE}

Reinhard MÜLLER, Menschenfalle Moskau. Exil und stalinistische Verfolgung. Hamburg, Hamburger Edition HIS Verlag, 2001, 501 p.

1 «Moscou, piège aux hommes " - tel est le titre du nouvel ouvrage de Reinhard Müller sur l'exil soviétique des communistes allemands pendant la terreur stalinienne. Pour la plupart des émigrés allemands, qu'ils soient venus en URSS avant ou après la prise de pouvoir par les national-socialistes, l'exil en URSS fut en effet un piège dont ils ne sortiraient plus jamais. Les quelques survivants constituèrent plus tard l'élite politique de la jeune République Démocratique d'Allemagne. Loin d'avoir échappé aux influences de l'environnement, semble dire Müller, ce groupe s'était constitué sur la base d'un concours de mensonge, d'infamie, de dénonciation et de perfidie. C'est le séjour à Moscou en tant qu'école de l'« inquisition stalinienne » vis-à-vis des émigrés politiques qui fait l'objet du livre.

2 À partir de nombreuses sources d'archives nouvelles, dont les archives du NKVD-MVD, Müller écrit l'histoire de la persécution du prétendu centre trotskiste d'espionnage Hoelz-Wollenberg qui, selon le NKVD, aurait réuni environ 70 émigrés politiques allemands en URSS - pour organiser des assassinats politiques contre les leaders communistes, planifier des actes de sabotage, rétablir le capitalisme, collaborer avec les hitlériens, etc. Bref, ce centre aurait été le groupe contre-révolutionnaire allemand, dans le démantèlement duquel était impliqué pratiquement tout l'establishment de l'émigration communiste allemande, que ce soit dans un rôle d'accusateur ou d'accusé, souvent avec un glissement du premier vers le second. Selon Müller, il n'est pas seulement question de révéler l'univers mental de l'informateur, avec «ce mélange inextricable de religion politique, de conception du monde intériorisée, d'animosité 
personnelle et de crainte». Il s'agit aussi de recontextualiser sur un plan microhistorique le processus réciproque de dénonciation, de stigmatisation et de sélection d'une part, et le système de pouvoir stalinien de l'autre, c'est-à-dire de montrer l'interaction entre institutions et individus - que les particuliers agissent selon leurs compétences fonctionnelles, prescrites par le système, ou bien en transgressant leur propre rôle et en suivant un principe universel, revendiqué ou non par le discours officiel. Ainsi les cas présentés varient largement, de la courageuse Frieda Düwell qui, malgré l'interdiction de contact avec les proches des inculpés, aida bien des familles frappées par les arrestations, jusqu'au délateur zélé Hans Schiff qui, soupçonnant trahison et contre-révolution partout, adressa volontiers ses signaux aux « organes ", même contre son propre frère.

3 La mise en rapport de la terreur d'en haut, des directives du NKVD ou des instances du Komintern, avec l'initiative personnelle, la contribution concrète d'en bas, sert de point d'appui à l'analyse de « la logique irrationnelle de la terreur». Pour cela Müller choisit une approche biographique. Dans sa reconstitution des parcours individuels, il se laisse guider en premier lieu par les dossiers du NKVD qui montrent très bien l'intérêt principal du pouvoir : découvrir et retracer les liens des inculpés entre eux pour élargir les réseaux de la prétendue conspiration et établir le principe de la «culpabilité par association» (guilt by association, cf. Hannah Arendt). Souvent ce sont même des connaissances éphémères ou bien des amitiés relevant d'un passé lointain qui font l'objet de l'interrogation, qui sont réinterprétées comme des liens politiques et servent de fondement à l'accusation, souvent avec le concours de «moyens physiques» (selon le jargon euphémisé des employés du NKVD).

4 Le "groupe Hoelz-Wollenberg ", dont les " enquêtes » établirent l'existence au fur et à mesure, comprenait en grande partie des membres de l'appareil militaire du parti communiste allemand. Nés dans les années 1890 et soldats volontaires de 1914-1918, ceux-ci se tournèrent vers le communisme sous l'impact de leur expérience de la Première Guerre mondiale. Ils luttèrent presque tous en faveur de la république des soviets à Munich en 1918 et furent par la suite emprisonnés dans la forteresse de Niederschönenfeld en Bavière - avec le célèbre anarchiste et poète Erich Mühsam (lui aussi activiste de Munich) qui périra en juillet 1934 après 17 mois de torture dans le camp de concentration nazi d'Oranienburg. Déjà en 1918, les cercles dirigeants du parti communiste se tenaient à l'écart de la république de Munich où d'autres partis, souvent anarchistes, exerçaient un rôle dominant. Toujours en prison, les communistes firent front contre les cercles anarchistes et contre Erich Mühsam qui, de son côté, pratiqua largement la polémique et eut une attitude critique envers les communistes, exacerbée avec le renforcement du règne de Stalin en Union soviétique. Plus tard, c'est sa veuve, Zenzl Mühsam, soucieuse de garder la mémoire et l'œuvre de son mari, femme simple et directe, qui se retrouva, une fois émigrée en Union soviétique, arrêtée et accusée aux côtés de communistes, ex-codétenus et adversaires idéologiques de son époux, dans un pays où elle n'avait choisi l'asile qu'à contrecœur, et où elle se trouvait dans une situation personnelle précaire. Il fallut beaucoup d'insistance de la part de la MOPR (Meždunarodnaja organizacija pomošči borcam revoljucii) et la promesse de publier les manuscrits de son mari pour la faire changer d'avis. C'étaient ces manuscrits qui rendaient Zenzl Mühsam intéressante aux yeux des communistes: juste après leur obtention, suivie de leur dépouillement par la section politique du NKVD et la rédaction 
d'un inventaire par l'Institut Marx-Engels, Z. Mühsam fut arrêtée pour la première fois (en avril 1936).

5 Déjà dans un livre antérieur, Die Akte Wehner (Le dossier Wehner, Berlin, 1993) - portant sur la période moscovite (1937-1941) du communiste Herbert Wehner, qui allait par la suite remplacer Willi Brandt à la tête du parti social-démocrate en Allemagne de l'Ouest -, Müller s'était intéressé au procès de Zenzl Mühsam, dans lequel Wehner avait joué un certain rôle. Dans les années 1920, Wehner était proche des cercles anarchistes allemands et admirateur d'Erich Mühsam, avec lequel il se brouilla après sa conversion au communisme. Son passé le rendait ainsi vulnérable et prêt à une collaboration inconditionnelle. Müller a découvert des documents prouvant que Wehner était loin de donner ses renseignements au NKVD sous la contrainte, ainsi qu'il l'affirme dans ses mémoires. Dans une lettre de février 1937, après la libération de prison (qui sera de courte durée) de Zenzl Mühsam sur intervention de Elena Stasova (chef de la MOPR), c'est Wehner qui prend l'initiative de s'adresser au NKVD pour offrir ses services. Il croit qu'il y a encore des liens, ne serait-ce qu'indirects, entre Mühsam et le trotskiste Wollenberg à Prague. Mais dans la mesure où Mühsam, se méfiant de lui, ne lui en aurait certainement pas parlé, il propose de s'infiltrer dans le club des ouvriers étrangers - « ce que j'ai évité jusqu'ici pour des raisons liées à la conspiration ", " où beaucoup de gens me connaissent et où je connais le passé de beaucoup de gens ». Cela lui aurait permis de " découvrir d'autres traces » et de révéler des « rapports avec l'ennemi ».

6 L'« ennemi » Erich Wollenberg, tête du groupe contre-révolutionnaire présumé, y était le seul trotskiste actif, mais il semble qu'il ne le soit devenu qu'après sa fuite d'Union soviétique pour Prague en 1934, où il fut logé et nourri par Zenzl Mühsam jusqu'au moment où elle rompit avec lui, dégoûtée qu'il eût utilisé son appartement pour ses orgies homosexuelles. Ils n'avaient donc pas eu de contact depuis plus de six mois lorsque Mühsam accepta finalement l'invitation de la MOPR en été 1935. Exilé en 1924, Wollenberg avait passé dix années en Union soviétique, ponctuées de courts séjours en Allemagne, où il fut blessé en juin 1932 par la SA après son intervention lors d'une réunion nazie, accident qu'il imputa au Comité central du parti communiste allemand, l'accusant d'avoir mal assuré sa protection. Le mécontentement vis-à-vis à la direction du parti s'accrut avec la prise de pouvoir par les nazis, quand nombre de communistes allemands eurent du mal à comprendre une ligne officielle qui ne voyait dans le règne nazi qu'un revers éphémère et continuait à considérer les sociaux-démocrates comme les adversaires principaux tout en accusant de défaitisme les communistes qui préconisaient un changement de stratégie.

7 C'est précisément dans ce contexte de désorientation des communistes allemands qu'une soirée dans l'appartement de l'ingénieur Taubenberger, le 5 mars 1933 (un certain nombre d'émigrés politiques allemands, dont Wollenberg, s'étaient rassemblés pour écouter les nouvelles des élections du Reichstag sur une chaîne allemande) constitua le point de départ de suspicions et de dénonciations qui devaient aboutir pendant la Grande Terreur à des accusations grotesques contre le « centre contre-révolutionnaire » et qui coûtèrent la vie à la plupart des participants. Une semaine environ après la réunion, Karl Schmidt, ex-déviationniste de la «droite brandlérienne » et réprimandé pour avoir utilisé l'interprétation de Kautsky dans une édition populaire du Capital de Marx, lance la dénonciation initiale dans une lettre à Fritz Heckert, représentant du PC allemand au sein du Comité exécutif du Komintern (IKKI). Il y accuse Wollenberg d'avoir critiqué le Comité central du PCA et affirme que Werner Rakow (pseudonyme: Felix Wolf), proche 
de Karl Radek et ancien membre de l'opposition de gauche, qui avait abjuré son hérésie en 1929 dans la " déclaration des 38 ", aurait accueilli avec joie l'arrestation du leader du PCA Ernst Thälmann par les nazis. Müller suppose que la délation fut écrite sous la pression de Heckert, auprès duquel Schmidt, membre des sociaux-démocrates tchécoslovaques, venait de déposer une demande d'autorisation de départ en Tchécoslovaquie. Lors de plusieurs séances de commissions d'enquête, auxquelles n'était toujours conviée qu'une partie des participants à la soirée, la Commission de contrôle du Komintern (finalement chargée de l'affaire) qualifia Rakow et Wollenberg - qui se défendaient avec véhémence contre l'accusation - d'ennemis de classe objectifs et annonça publiquement dans deux journaux communistes germanophones leur exclusion des rangs du parti. Cette décision, accompagnée de reproches contre les autres participants de la soirée, était censée remettre au pasles communistes allemands en Union soviétique et ailleurs. Elle ne manqua pas d'effet et toute une vague de critiques et d'autocritiques s'ensuivit.

8 À la différence de Wollenberg qui, en arrivant à Prague, se rapprocha vraiment des trotskistes, devint un proche collaborateur de Léon Sedov et commença à publier des articles contre la dictature de Stalin dans des journaux trotskistes, Max Hoelz, l'autre pilier du "centre contre-révolutionnaire Hoelz-Wollenberg ", "se noya " (selon la version officielle) en été 1933 dans l'Oka près de Nijni-Novgorod. Arrivé en Union soviétique en 1930 et célébré comme un héros de la classe ouvrière, il s'était rapidement heurté à "l'esprit de caserne", on lui avait reproché son manque de discipline, son comportement impulsif, son anarchisme et sa vie privée (notamment ses relations sexuelles). Il avait écrit de nombreuses lettres aux leaders politiques pour obtenir une autorisation de quitter l'URSS, en se plaignant des contraintes qu'il subissait ainsi que des accusations portées contre lui. Mais, condamné à rester en URSS, il tomba bientôt dans un état de forte dépression, s'enferma dans sa chambre d'hôtel en soupçonnant tout son environnement - de la femme de ménage jusqu'au garçon de la cantine - de l'espionner sur ordre de la GPU. Complètement désespéré, il finit par s'adresser en mars 1933 à un employé de l'ambassade allemande et lui demanda de l'aide pour obtenir une autorisation de quitter l'URSS (qui lui fut refusée), acte qu'il regretta immédiatement, sachant que l'ambassade était sous surveillance permanente et que le contact avec les représentants du régime nazi était considéré comme une forme de désertion. Il se repentit en dénonçant Wollenberg - opération visiblement arrangée entre eux - et partit travailler dans un kolkhoze de province où sa mort fut très vraisemblablement l'œuvre d'agents du NKVD, acte sur lequel on n'a jamais retrouvé trace dans aucun document.

Si, vis-à-vis des « organes ", Hoelz prend ses distances par rapport à Wollenberg dans une lettre adressée au Département des cadres, à peu près au même moment il en envoie une copie directement à Wollenberg où il rajoute quelques lignes pour lui exprimer son estime. Ils se rassurent donc mutuellement et en secret sur leur amitié. En revanche, chez beaucoup d'autres, la séparation d'avec leurs amis signifie une désolidarisation bien plus brutale et définitive. Quand Taubenberger, par exemple, rédige en mai 1933 une requête à la Commission de contrôle pour revendiquer l'exclusion du parti de Hoelz dont «le cadavre commence à puer », ce comportement, qui n'est pas associé à une complicité secrète, provoque la rupture des liens personnels, indépendamment de la motivation concrète du dénonciateur.

10 Après le meurtre de Kirov, les répressions prennent de l'ampleur, y compris contre les Allemands. Müller parle d'« archives de l'inquisition» pour les dossiers des cadres qui 
servent à fouiller dans le passé de chaque communiste. Celui-ci, ainsi que les hérésies de jadis, compte de plus en plus et crée une atmosphère où il n'y a bientôt plus de parole sans arrière-pensée, où les mots deviennent captieux à la lumière des biographies des interlocuteurs. De plus en plus de communistes allemands y contribuent par des dénonciations, souvent quand ils se sentent eux-mêmes en danger à cause d'un point faible dans leur parcours, ou bien pour leur «lien» avec des gens démasqués comme "éléments nuisibles»: manifester de la «vigilance» semble le seul moyen de s'accréditer auprès du parti. En dépit de la cruauté de cette "inquisition", les exclusions, les arrestations, etc., semblent d'une certaine façon plausibles pour un grand nombre de «bons croyants» (ici Müller renvoie aussi aux mémoires des survivants), qui ne commencent à perdre leur foi qu'au moment où les vagues d'arrestations finissent par emporter - à côté des critiques de Stalin - des gens qui s'étaient toujours distingués par la fidélité avec laquelle ils soutenaient et défendaient la politique et les slogans du régime. Au début, on parle d'erreurs de la part des organes, selon Müller " pour ne pas mettre en danger son identité politique, son "moi-parti" et le mythe de Stalin. » Mais plus tard, quand les têtes commencent à tomber partout, la peur brute prend le dessus. Selon les mémoires des survivants, presque personne n'aurait osé imaginer jusqu'au bout les conséquences de ce qui se passait et en tirer la conclusion que Stalin lui-même était l'initiateur de la terreur. Cependant, comme les Soviétiques, les Allemands n'arrivent pas non plus à sortir de la « fiction totalitaire », des « fantasmes du soupçon généralisé », et recourent à des théories du complot, selon lesquelles les agents des ennemis, fascistes, espions, etc., se seraient infiltrés dans les organes du NKVD et essaieraient de saigner la société soviétique de l'intérieur - à l'insu de Stalin.

11 À côté d'un grand nombre de procès-verbaux d'interrogatoires à la Lubjanka, qui montrent bien les comportements individuels, mais n'aboutissent pas à une typologie convaincante (les cas présentés ne sont pas utilisables sur un plan catégoriel: "Le dénonciateur: Hans Schiff», "L'homosexuel : Peter Holm», "La tête carrée: Herbert Berndt »), Müller présente aussi les ordres du NKVD concernant les Allemands et la manière dont ils sont exécutés. L'ordre $n^{\circ} 00439$ (25 juillet 1937), qui au début ne prévoit que l'arrestation des Allemands travaillant dans l'industrie d'armement, est de plus en plus appliqué à tous les «Allemands » : aux émigrés politiques, citoyens soviétiques dans la république de la Volga et même à des personnes ayant un nom à consonance allemande. La lettre circulaire $\mathrm{n}^{\circ} 68$ du 22 août 1937 déclare que la grande majorité des étrangers en URSS sont des espions et des " diversionnistes »; pour y remédier, elle commande de ne prolonger aucun visa, à l'exception des ressortissants d'une petite liste de pays (l'Allemagne n'en fait pas partie), qui ne doivent être expulsés qu'au cas où on disposerait de documents compromettants contre eux. Néanmoins, à en juger par les chiffres et les parcours présentés par Müller, le cas de l'Autrichienne olga Meese qui, après une condamnation à 10 ans en juillet 1937, est expulsée en 1940 en Allemagne avec 12 autres détenus, semble plutôt exceptionnel. Meese venait régulièrement voir son ami Alberto Zenari, Italien invalide et soi-disant membre du « centre trotskiste » à Moscou, elle fut accusée de rendre des services de courrier pour Wollenberg. En pratique, et dans la mesure où l'arrestation est préférée pour neutraliser « les espions ", l'ordre d'expulsion forcée des étrangers n'a d'effets que pour la présélection des demandeurs d'asile et rend encore plus restrictive la politique d'accueil, déjà marquée par le soupçon généralisé, et dont Müller décrit les processus et les critères en détail. On soupçonne particulièrement les évadés ou libérés des camps de concentration nazis : souvent ils passent d'un enfer à l'autre, comme Werner Hirsch qui est «chaleureusement » accueilli à Moscou par un procès, à propos 
duquel Zenzl Mühsam à Prague écrit dans une lettre interceptée par l'appareil du PCA : « Dis donc, Werner, est-il vrai que tu aies besoin de t'excuser d'avoir survécu au camp?». En 1936, le lien entre Hirsch et Mühsam permet au NKVD de le compter parmi les contrerévolutionnaires du centre Hoelz-Wollenberg, bien qu'il ait rencontré Mühsam sur ordre du parti pour la persuader de venir à Moscou avec les manuscrits de son mari.

Le destin ultérieur de Zenzl Mühsam, héroïne tragique, constitue l'épilogue du livre: deuxième puis troisième arrestation, refus catégorique de faire des aveux comme le souhaitent les enquêteurs, puis expulsion (1955) en RDA, où elle est mise sous surveillance permanente de la Stasi. Une semaine avant sa mort, dans un état de forte dépression, elle finit par céder ses droits sur les manuscrits de son mari - entre-temps microfilmés à l'Institut Gorki - à l'Académie des arts de Berlin-Est.

13 Alfred Kantorowicz, qui l'a rencontrée lors de son arrivée en RDA, note que le destin de Zenzl Mühsam et celui de milliers d'autres contribue à l'épopée du $\mathrm{xx}^{\mathrm{e}}$ siècle, qu'il sera peutêtre impossible à fixer par le biais de la littérature. Le récit documentaire serait vraisemblablement le seul genre approprié - conseil que Müller a mis en pratique - ce qui explique aussi qu'il fasse l'économie d'une conclusion. Outre l'aspect documentaire, les repères théoriques, notamment les analyses de Michel Foucault sur l'animal confessant et sur le parti comme panopticon, les réflexions de Elias Canetti sur le maniement du secret par le détenteur du pouvoir et avant tout l'étude classique de Hannah Arendt sur le totalitarisme, restent un peu sous-exposés, ce qui est visiblement dû à une indignation morale très compréhensible de l'auteur. N'est pas non plus tout à fait convaincante la cohérence sans rupture que Müller établit entre une « disposition wilhelmienne nationaliste autoritaire", qui se manifesterait par la participation volontaire à la Première Guerre mondiale, et « l'esprit de caserne » des « soldats du parti » disciplinés dans les années 1930 notamment dans la mesure où l'évaluation des années 1910 et 1920 est donnée uniquement sur la base des autobiographies « soviétiques » des années 1930 (où les protagonistes avaient évidemment intérêt à souligner leur discipline et leur vigilance) - et à la lumière des jugements parfois polémiques d'Erich Mühsam contre ses adversaires politiques. Certes, il est juste de renvoyer à l'expérience de la guerre qui a sans doute marqué toute une génération d'Européens. Par contre, pour rendre intelligibles les raisons du glissement de l'engagement envers une idée humaniste à une " obéissance de cadavre » (selon Müller), il faudra creuser plus profond, notamment pour les deux premières décennies du siècle, où la liberté de choix et de décision morale était plus grande que dans les années 1930 entre l'Allemagne nazie et l'Union soviétique stalinienne.

Mais le livre de Reinhard Müller porte sur les années 1930 et pour cette période la documentation est extrêmement riche et bien présentée. Un index et une liste des victimes avec des aperçus biographiques facilitent l'orientation dans la multitude des noms allemands. C'est une mine à découvrir non seulement pour qui travaille sur l'exil, mais aussi pour tous ceux qui s'intéressent au stalinisme tout court et à la " préhistoire " de la RDA. 